\title{
PREDICTIVE CONTROL FOR ENERGY MANAGEMENT IN ALL/MORE ELECTRIC VEHICLES WITH MULTIPLE ENERGY STORAGE UNITS
}

\author{
M. J. West C. M. Bingham N. Schofield \\ Electrical Machines and Drives Group, Department of Electronic \& Electrical Engineering, University \\ of Sheffield, UK
}

\begin{abstract}
The paper describes the application of Model Predictive Control (MPC) methodologies for application to electric and hybrid-electric vehicle drive-train formats incorporating multiple energy/power sources. Particular emphasis is given to the co-ordinated management of energy flow from the multiple sources to address issues of extended vehicle range and battery life-time for allelectric drive-trains, and emissions reduction and drivetrain torsional oscillations, for hybrid-electric counterparts, whilst accommodating operational constraints and, ultimately, generic non-standard driving cycles.
\end{abstract}

\section{INTRODUCTION}

The drive towards the development and realisation of environmentally friendly vehicles is resulting in the adoption of new power train formats, augmenting the more traditional internal combustion engine (ICE) with electrical torque assist, or with multiple electrical energy/power sources. Although significant advances are occurring in battery and fuel-cell technologies to improve their energy density and cyclic efficiency/lifetime, due to their limited specific power capability, alternative peak-power buffer technologies, viz. super-capacitors and high-speed flywheels, are being developed for incorporation into the drive-train. A primary objective is, therefore, to improve both the vehicle range and battery cycle life through optimal management of the power and energy, and the full utilisation of installed capacity.

With the increasing complexity of power train formats, however, comes the requirement for more advanced control strategies to extract maximum benefit from each energy source. Here, the paper discusses the application of predictive control techniques for enhancing battery-lifetime and vehicle range, and reducing emissions and drive-train oscillations by integrated torque assist techniques.

\section{A. Classical energy management techniques}

Methodologies currently employed for energy management are generally the result of extensive experimental trials and iterative modifications, resulting in map-based empirical solutions that possess limited flexibility to cope with different driving styles and driving cycles or the real-time macrodynamics of the power-train, which can have a significant effect on the long-term economic utilisation of the energy storage components.
The design of engine control systems has similarly been traditionally carried out using a blend of heuristic techniques validated by simulations and prototyping using approximate average-value models. Increasing demands from emissions legislation, however, calls for more robust techniques [1].

The complementary management of mechanical and electro-magnetic braking can impact on the performance of other vehicle control systems such as anti-lock braking (ABS) and traction control (TC), where transient torque control to the driven wheels is used to optimise slip between the $\mathrm{road} /$ tyre interface. To-date, a feature of many ABS/TC systems for commercial IC-engine vehicles, is that the realisable performance is often limited by the bandwidth of electro-hydraulic actuation systems. However, with the advent of electrically powered vehicles into the marketplace, the means for controlling wheel drive-torque at much greater bandwidths is possible, with for example, the mechanical brakes providing a low bandwidth mean braking torque and the electromagnetic counterpart imposing the high bandwidth torque transients.

\section{B. Benefits of enhanced energy management}

Whilst it is relatively straightforward to establish optimal energy management schemes for standard driving cycles, real driving conditions will invariably result in significantly different performance, with the probability that transient battery currents will compromise both achievable range and cycle life. Fundamental issues to be addressed in order to achieve optimal energy utilisation over wide ranging operating conditions are generally applicable to many powertrain formats, and include:

- The dynamic apportioning of the energy requirements between different energy storage/conversion units.

- The incorporation of economic factors, such as the impact of dynamically varying charge/ discharge profiles on the cycle life of the battery, and the battery replacement cost, into the overall energy management strategy.

- The incorporation of environmental factors, such as reducing emissions due to transient throttle operation of internal combustion engine.

- The accommodation of deviations from the optimal operating point for each energy storage unit so as to promote high efficiency. 


\section{MPC for energy management}

Over recent years, various MPC techniques have been devised and employed in a range of complex industrial and process control applications [2,3], a common feature being its application to problems involving plants with multiple inputs and outputs, and those with strict economic, actuator or safety constraints. The widespread application of MPC within such fields as chemical and process industries is indicative of its potential, and, whilst computationally expensive, it is anticipated that the advent of explicit solutions to the Quadratic Programming sub-problem, from efficient algorithms such as Multi-Parametric Quadratic Programming [4], will facilitate the increasing spread of MPC to highbandwidth systems. It is therefore considered timely to investigate the utilisation of MPC for energy management in al/more-electric vehicles, with the added flexibility to naturally scale solutions to multi-input-multi-output systems, and the inherent ability to handle hard and soft constraints in a unified, stable manner. Indeed, constraint management, imposing specific bounds on inputs/outputs/states, is employed to provide safety limitations, satisfy environmental regulations and physical restrictions. Although careful tuning of a classically designed controller may keep variables away from the pre-specified bounds, ideally, the control system should drive the process as close as possible towards the constraints without violating them, since, in general, this maximises the performance (and often economy) $[2,3]$.

Constrained MPC therefore employs a more direct approach to constraint management, by modifying optimal unconstrained solutions in a manner that the constraints are not violated. Here, some of the benefits arising from the ability of MPC to accommodate constraints on input, output and system states, are considered for the separate cases of respectively, an all electric drive-train, and a mild hybridelectric vehicle.

\section{ALL-ELECTRIC DRIVE-TRAIN}

\section{A. Problem description}

An example all-electric vehicle drive-train is shown in Fig.1(a), where the peak power buffer is typically connected directly to the traction drive, with the dc-link voltage being allowed to vary continually, whilst the $\mathrm{dc} / \mathrm{dc}$ converter has only to provide the average vehicle power. To date, proposed energy management schemes for such drive-trains have been relatively simple, in that power is drawn directly from the battery whenever the dc link voltage falls below a minimum specific level, whilst regenerative energy is diverted to the battery if the peak power buffer becomes fully charged.

\section{B. Formulation of control problem}

Methodologies currently employed for energy management in electric vehicles are generally the result of extensive experimental trials and iterative modifications, resulting in map-based empirical solutions that have limited flexibility to cope with different driving styles and driving cycles $[5,6,7]$, or the real-time macro-dynamics of the power-train that can have a significant effect on the long-term economic utilisation of components. Given the complex multiobjective performance/economic dynamics of these systems, it is highly likely that optimal management schemes will ultimately incorporate short-term adaptation mechanisms to accommodate different driving profiles, with longer-term adaptive schemes to facilitate minimal component wear/cost.

\section{MPC for an all-electric drive-train}

A basic MPC methodology to control the power drawn from a battery pack and a super-capacitor peak power buffer, to supply an all-electric drive-train, is presented, that inherently accommodates the total stored energy in the system in real-time to reduced mechanical braking effort, and hence, brake wear. In addition, the recovery of regenerative energy allows improvements in overall drive-train efficiency. By way of example, Fig.1(a) shows an example all-electric drive-train format, whilst Fig.1(b) shows a simple model describing the dynamics of power transfer and distribution. To focus the study on the control of energy, internal resistances of the super-capacitors (and their associated interconnects) and thermal considerations, have been omitted. The nominal drive-train is considered to consist of a $45 \mathrm{~kW}$ permanent magnet brushless DC traction machine supplied from a $300 \mathrm{~A}, 600 \mathrm{~V}$ IGBT inverter. The super-capacitor banks each consist of $50 \times 2500 \mu \mathrm{F}$ series-connected capacitors, totalling $C_{s c}=50 \mathrm{~F}$, having a maximum voltage of $135 \mathrm{~V}$. The step-up $\mathrm{dc} / \mathrm{dc}$ converters are each rated at $33 \mathrm{~kW}$ with a maximum output voltage of $150 \mathrm{~V}$.
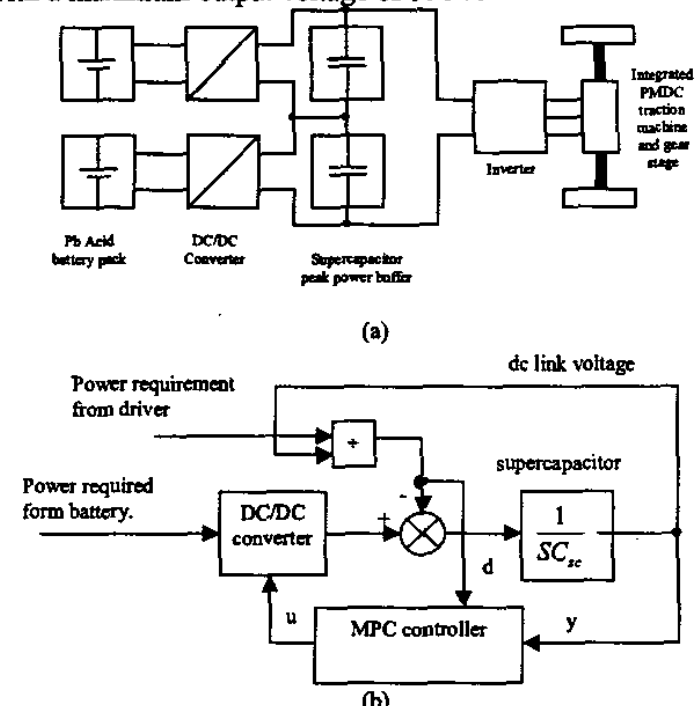

Fig. 1 Electric vehicle drive train format and power flow.

The system control requirement in this case is to maintain the output voltage of the peak power buffer within required limits whilst ensuring uni-polar battery current (no 
regeneration) and minimising the battery current magnitude. The driving cycle, which is assumed to be unknown to the vehicle controller, constitutes a power disturbance to the system. A variant of MPC employing zone control $[2,8]$ is adopted as being particularly attractive for this application. field.

Net energy expenditure is the normal requirement for most driving conditions, and results in an overall net outflow of energy from both the peak power and the primary energy source (in this case the lead acid traction battery pack). Since it is necessary to penalise the magnitude of current transferred directly to/from the battery pack, there is a tendency for the output voltage of the peak power buffer to approach the lower limit of the controlled 'zone'. However, when descending a gradient, for example, the net energyexpenditure requirement can reduce to zero or become negative. In this case, the peak power buffer is charged towards the upper limit of the zone, with the controller acting to remove energy from the peak power buffer only when the output voltage exceeds the upper dc-link voltage limit, thus recharging the battery. In instances when the cells are fully charged, and hence, cannot accept additional charge, or, when the vehicle primary energy source does not allow for regeneration viz. IC engine hybrid or fuel-cell vehicle, energy dissipation is realised by either resistive 'dumping' or the application of mechanical brakes.

The key difference between the proposed strategy and classical energy management strategies described previously, that act to maintain the dc-link voltage about a pre-defined set point, is that any deviation above the set-point, whether as a result of short term regenerative braking effort or net energy gain over a longer period, would be counteracted with a negative control action to remove energy from the peak power buffer even when excess storage capacity still exists. Although, ultimately, both strategies draw their energy from the primary energy storage unit to accommodate a given driving cycle, the simple dc-link voltage control methodology unnecessarily causes more cycling of energy to/from the battery pack, or requires more mechanical braking to dissipate the energy. Hence, employing MPC, with its inherent ability to include zone control, will generally result in higher overall drive-train utilisation efficiency.

\section{Zone Controller}

In common with other MPC strategies, the zone controller minimises a cost function, $J$, in the presence of constraints, over a fixed prediction horizon (1). This form of cost function is characteristic of Generalised Predictive Control[9] and consists of the squared deviation of the predicted output voltage, $\hat{y}$, from the reference trajectory, $r$, and the weighted squared increment of control action, $u$. Constraints are imposed on inputs, outputs or states eg. control actuator limitations such as slew rate, and are represented here in a generalised form:

$$
\begin{aligned}
J(u, k) & =\sum_{j=N c}^{N}(\hat{y}(k+j \mid k)-r(k+j))^{T}(\hat{y}(k+j \mid k)-r(k+j)) \\
& +\lambda^{2} \sum_{j=1}^{N} \Delta u^{T}(k+j-1) \Delta u(k+j-1) \\
& \text { subject to the linear inequality constraints } \\
\tilde{\psi}(k) \leq \tilde{\Psi}(k) &
\end{aligned}
$$

where $N_{c}>1$ is the control horizon, which is the prediction period for the control action; $N$ is the prediction horizon, which is the period over which the dynamics of the system are predicted at each sample step; $\hat{y}(k+j \mid k)$ is the prediction of output voltage at time $k+j$, given knowledge up to time $k, \Delta u(k)$ is the increment in the control signal demand at each sample step; $\lambda \in \mathbf{R}$ is the control weighting factor; $\tilde{\psi}(k) \leq \widetilde{\Psi}(k)$ specify the constraints on inputs, output and states [2] e.g. $\tilde{\psi}(k) \leq \widetilde{\Psi}(k)$ might include $\Delta u(k)<5$, which limits the slew rate of the control action to be less than $5 \mathrm{~A} / T_{s}$, where $T_{s}$ is the sampling period of the controller.

Accommodating a controlled 'zone', about which the output voltage of the peak power buffer is allowed to vary, is included by appending an additional 'slack' variable $\delta(k)$ into the GPC cost function:

$$
\begin{aligned}
J(u, k) & =\sum_{j=N c}^{N}(\hat{y}(k+j \mid k)-r(k+j)+\delta(k+j))^{T}(\hat{y}(k+j \mid k)-r(k+j) \\
& +\delta(k+j))+\left\{\lambda^{2} \sum_{j=1}^{N} u^{T}(k+j-1) u(k+j-1)\right\} \\
& \text { subject to the linear inequality constraints } \\
\tilde{\psi}(\mathrm{k}) & \leq \widetilde{\Psi}(\mathrm{k}) \& \\
|\delta(k)| & \leq \delta_{\max }
\end{aligned}
$$

where $u(k)$ is the control signal, update every sample step, and $|\delta(k)| \leq \delta_{\max }$ is an additional constraint on the slack variable (note: more details on this formulation can be found in $[2,8])$. The additional slack variable allows the output voltage of the peak power buffer to deviate from the nominal reference value by a predetermined amount, $\delta_{\max }$, before it contributes to the cost function. This then, 'frees' the peak power buffer from the requirement of trying to control a nominal output voltage. Although other control methodologies can be 'de-tuned' to provide this characteristic, predictive control techniques readily provide an integrated framework to accommodate this feature in an optimal manner.

To develop the MPC strategy, the model shown in Fig.1(b) can be represented by process and disturbance dynamics (which constitute the current requirements from the driving cycle), see Fig.2: 


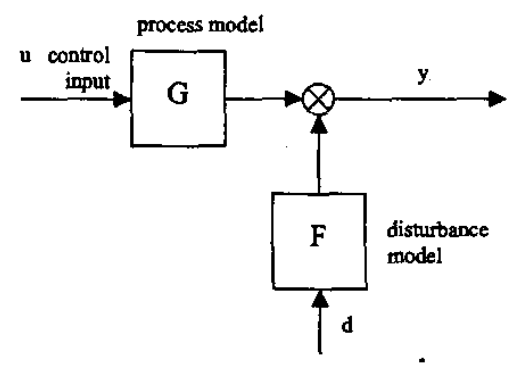

Fig. 2 Process disturbance dynamics

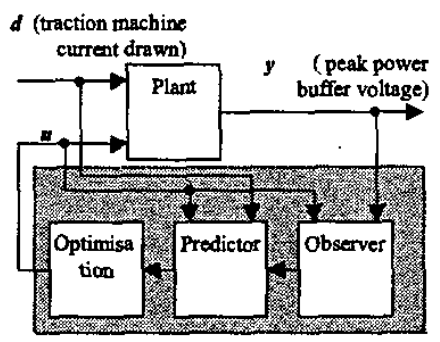

Fig. 3 Controlles

where $G=-F=\frac{1}{S C_{s c}}$ and is described in state-variable form by:

$$
\begin{aligned}
& G \Rightarrow \dot{x}_{G}=0 x_{G}+u_{G}, \quad y=1 / C_{s c} x_{G} \\
& F \Rightarrow \dot{x}_{F}=0 x_{F}-d, \quad y=1 / C_{s c} x_{F}
\end{aligned}
$$

Calculation of the control action at each sampling step, subject to the constraints, is well documented $[2,3,8]$. However, the underlying principles are to minimise the cost function, $J(u, k)$, over an $N$-step look-ahead horizon from the discretized dynamics of (3).

\section{E. Results}

To demonstrate the benefits of the proposed methodology, and its impact on electrical vehicle braking systems, the power requirements of the standard ECE15 driving cycle, is calculated from the vehicles dynamics to incorporate a negative $1.53 \%$ downhill gradient of the road. It is interesting to note that this reduces the mean power required to satisfy the nominal driving cycle requirements by $50 \%$.

A comparison of controller induced energy flow dynamics, for the regulation of the peak power buffer voltage around the nominal operating point (classical), and the proposed MPC controller with zone control, are illustrated, respectively, in Fig.4 and Fig.5; the DC link voltage, current drawn by the traction inverter and by the DC/DC converter into the peak power buffer, simulated over $2 \times$ ECE15 cycles, with a fixed $-1.53 \%$ gradient. The prediction horizon is selected to be $N=16$, i.e. predicting 8 seconds ahead at each sample time, and a cost function weighting factor of $\lambda=1.8$ is employed to provide a compromise between accommodating disturbance rejection and the magnitude of resulting control action. From the results, it is seen that there is a significant reduction in the peak currents required from the battery pack, in both cases, by virtue of employing the peak-power buffer. However, in the case of the MPC 'zone' control, Fig.5, no reverse energy flow from the buffer is apparent, whereas a negative energy flow is often required from the peak power buffer to maintain the nominal output voltage in the case of employing voltage control, Fig.4.

It is also noted from Fig.4 and Fig.5 that, whilst the realtime power requirements is the same for both techniques, differences in the current requirements to the traction machine from the peak power buffer, are apparent.

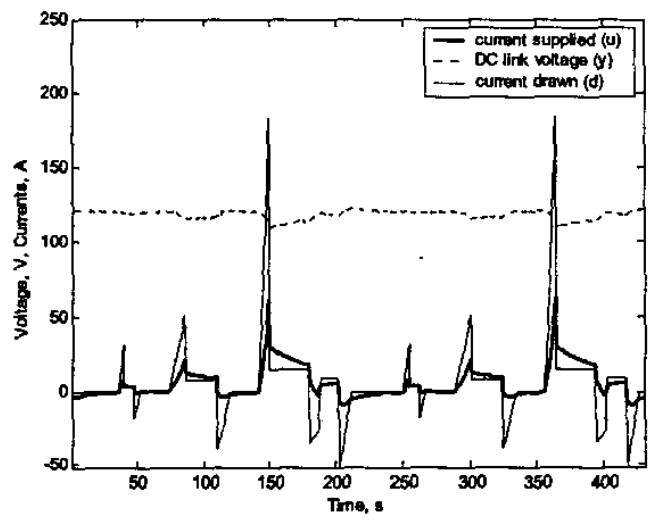

Fig. 4. Non zone control

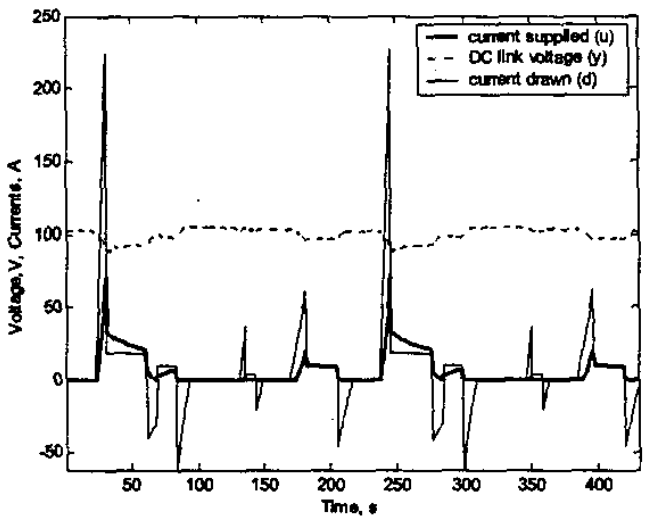

Fig. 5. Zone control

The amount of energy 'returned' to battery pack, resistively dumped or removed by mechanical braking, for both methodologies, is summarised in Table 1 . The results imply that no mechanical braking is required with ' $z$ one' control since all the braking requirements are accommodated 
by regeneration, in this case, irrespective of the ability of the primary energy source to accept charge, whether limited as a result of the choice of technology or state of charge of the traction battery. However, of course, this will by no means always be the case, as many driving situations present much more demanding disturbance profiles eg. emergency stop or stopping from higher speeds, as encountered on an ECE suburban driving cycle, for instance. Nevertheless, even under the most arduous braking envisaged, benefits of zone control will enhance the management of energy flow throughout the system.

TABLE I -

\begin{tabular}{|l|l|l|l|}
\hline Controller & $\begin{array}{l}\text { Energy drawn } \\
\text { from battery } \\
\text { /JJ }\end{array}$ & $\begin{array}{l}\text { Energy } \\
\text { returned to } \\
\text { battery } / \mathrm{kJ}\end{array}$ & $\begin{array}{l}\text { Net energy } \\
\text { expenditure } \\
\mathrm{kJ}\end{array}$ \\
\hline $\begin{array}{l}\text { MPC zone } \\
\text { control }\end{array}$ & 262 & 0 & 262 \\
\hline $\begin{array}{l}\text { dc-link voltage } \\
\text { control }\end{array}$ & $318(121 \%)$ & $56(21 \%)$ & 262 \\
\hline
\end{tabular}

\section{HYBRID ELECTRIC DRIVE-TRAIN}

\section{A. Problem description}

Fig.6, illustrates a possible mechanical arrangement of a parallel torque assist drive train, where the power from an ICE is augmented with electromechanical torque from a motor/generator. Although diagrammatically, a 'beltcoupling' is shown, this is considered to represent any mechanism by which torque addition can be obtained. The two free control variables to tune the power split between the electrical machine and the ICE, is the torque that each produces, whilst their speeds are dictated by instantaneous wheel speed (although possibly with a different mechanical ratio). When a vehicle is subjected to acceleration or disturbances, the flexibility of the various components in the driveline is known to cause torsional vibrations which can result in transient oscillations in vehicle speed. Such driveline oscillations, known as shuffle, are dominated by the first resonance frequency of the driveline, giving rise to material fatigue stress and reduced vehicle driveability.

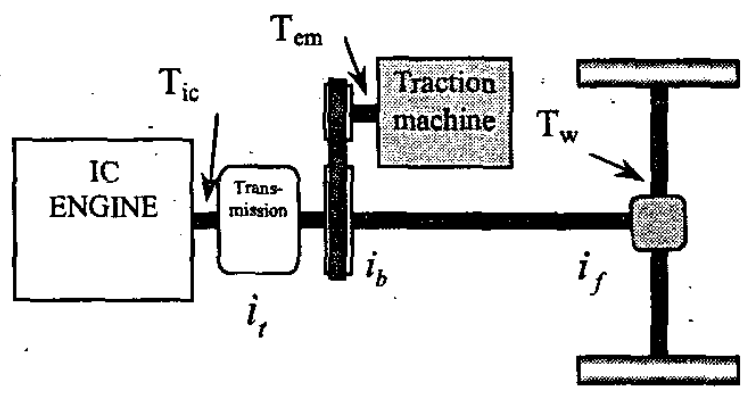

Fig. 6. Possible Hybrid drive train
It is proposed that the inherent ability to control systems with transport delays using MPC, [3] make it a primary candidate for torque control of both series and parallel hybrid vehicles, due to the requirement to accommodate the pure delays inherent to the four stroke internal combustion engine, see Fig. 7. The severe oscillations, also shown in Fig.7 could be minimised or eliminated by active torque control of both power sources, but only by taking direct account of the transport delay, drive-train stiffness and lash.

In addition to the reduction in transient throttle movements, which hamper emission control algorithms due to their low bandwidth, a predictive control model of the entire hybrid drive system allows shaping of the acceleration response (i.e. refine the transients seen in Fig.7), and reductions in the throttle lag and shuffle, leading to significant improvements in driveability.

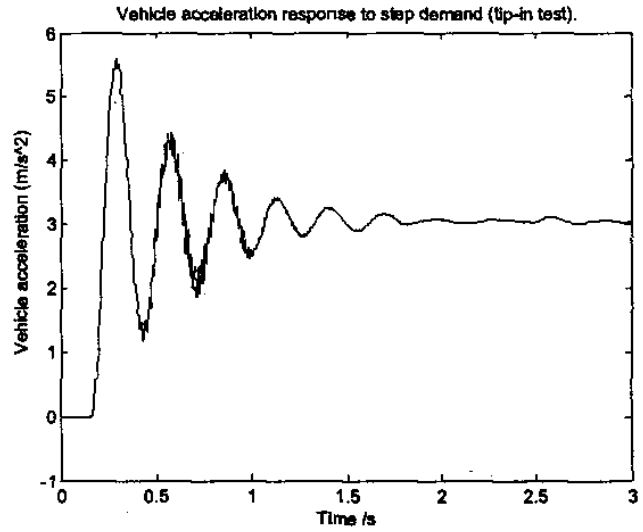

Fig. 7. Vehicle Acceleration response to step demand (tip in test)

\section{B. Classical control}

During transient operation, a spark ignition engine develops substantially higher emissions than during static operation. This is due to the imperfect adaptation of mixture formation during the transitional phases associated with dynamic operation. When the throttle valve opens abruptly, a portion of the fuel supplied by a throttle body fuel injection unit or carburettor, condenses and remains inside the intake manifold. These systems therefore rely on acceleration enrichment strategies for compensation.

A model of a port fuel injected spark ignition engine, as described in [10], simplified by linearising volumetric efficiency, pumping and friction losses, and with a fixed spark advance, provides typical throttle angle, Fig.8, and corresponding stochiometric air fuel ratio, Fig.9, dynamic characteristics. 


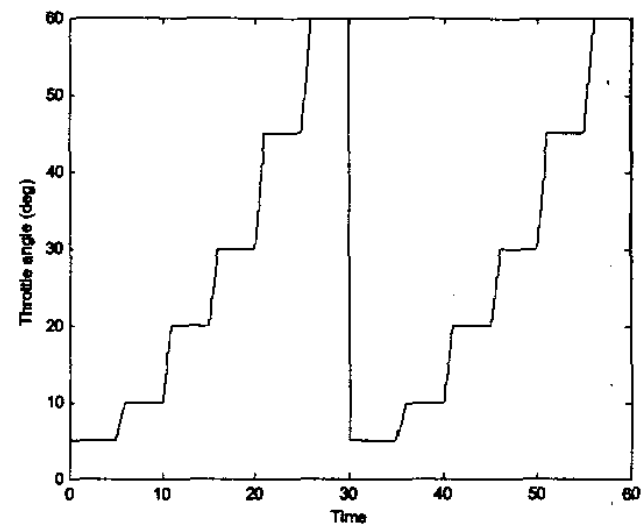

Fig. 8 Throttle position

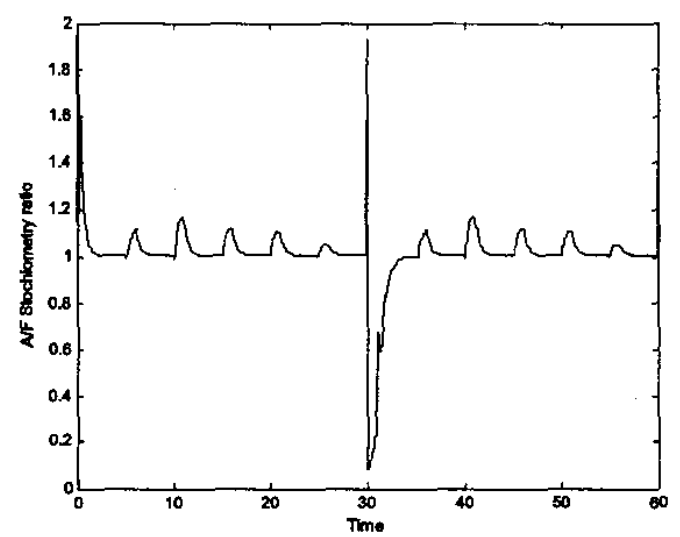

Fig. 9. Air fuel stochiometry ratio

This simple model shows the excessively lean mixture on a throttle opening transient, and an excessively rich mixture on a throttle closing transient. The fuel injector command is based on classical engine management strategies, from a look-up table of speed and air intake mass flow rate. The lambda sensor, when used in closed loop control provides only steady correction to the look-up table, with values often stored in non-volatile memory. Due to the sensor's position in the exhaust manifold, there is a significant delay of between 0.5 and 12 seconds [12], before the air-fuel ratio is determined. This effectively prevents use of the lambda sensor to control the air/fuel ratio for transients, and, instead, enrichment schemes are employed on detection of acceleration demands. To pass latest proposed legislation, it is likely that the control of such transients will need to be enhanced.

\section{Formulation of Control Problem}

A typical hybrid-drive-train consists of a clutch, propshaft, final drive, drive-shafts, wheels and tyres. The derivation of a model, employed here for the design of a model predictive controller, can be obtained in many ways, as a result of rigorous modelling of the entire system, or by more advanced system identification methods. Although there are many significant torsional resonances in the driveline, a linear model including a drive-shaft flexible mode is sufficient to show the measured engine speed and wheel speed. The derivation of such a model is straight forward and can be found, for example, in [13]. Although it is recognised that, high-order ICE dynamics should ultimately be modelled, to demonstrate the benefits of explicit constraint handling by predictive controllers, the ICE and electrical machine dynamics are considered here as static gains for simplicity.

The model of the vehicle drive train, presented in state space form in (4) \& (5), is coupled with the dynamics of the super-capacitor bank (3). For this application of constrained GPC, it is useful to employ incremental control signals, and, consequently, the actual inputs are considered as augmented states (7).

$$
\begin{aligned}
& \text { where } \\
& i=i_{t} i_{f}, \quad J_{1}=J_{m}+J_{t} / i_{t}^{2}+J_{f} / i_{t}^{2} i_{f}^{2}, \quad J_{2}=J_{w}+m r_{w}^{2} \\
& b_{1}=b_{t} / i_{t}^{2}+b_{f} / i_{t}^{2} i_{f}^{2}, \quad b_{2}=b_{w}+c_{w} A_{a} \rho_{a} r_{w}^{3}+m c_{r 2} r_{w}^{2} \\
& l=r_{w} m\left(c_{r 1}+g \sin (\alpha)\right)
\end{aligned}
$$$$
\begin{gathered}
\mathbf{A}=\left[\begin{array}{ccc}
0 & \boldsymbol{x}=\mathbf{A x}+\mathbf{B} u+\mathbf{H} l & 1 / i \\
-k / J_{1} & -\left(b_{1}+c / i^{2}\right) / J_{1} & c / i J_{1} \\
k / J_{2} & c / i J_{2} & -\left(c+b_{2}\right) / J_{2}
\end{array}\right] \\
\mathbf{B}=\left[\begin{array}{cc}
0 & 0 \\
1 / J_{1} 1 / J_{1} \\
0 & 0
\end{array}\right] \quad \mathbf{H}=\left[\begin{array}{c}
0 \\
0 \\
-1 / J_{2}
\end{array}\right] .
\end{gathered}
$$

The variables in (6) are defined in Table II.

TABLE II

DRIVE-TRAIN MODEL VARIABLES

\begin{tabular}{|l|l|}
\hline$J_{m}$ & Inertia of ICE and integrated electrical machine \\
\hline$J_{t}$ & Inertia of transmission \\
\hline$J_{f}$ & Inertia of final drive \\
\hline$i_{t}$ & Transmission ratio \\
\hline$i_{f}$ & Final-drive ratio \\
\hline$b_{t}$ & Transmission viscous damping coefficient \\
\hline$b_{f}$ & Final-drive viscous damping coefficient \\
\hline$b_{w}$ & Wheel viscous damping coefficient \\
\hline$c_{w}$ & Drag coefficient \\
\hline$A_{a}$ & Vehicie cross-sectional area \\
\hline$\rho_{b}$ & Density of air \\
\hline$r_{w}$ & Radius of wheel \\
\hline
\end{tabular}

The final multi-input multi-output model states, (7) correspond to the drive-shaft torsion, the wheel and engine angular velocities, the super-capacitor power buffer voltage, $V_{d c}$, and the controller outputs the torque demand to the ICE, $T_{i c}$ and the torque demand to the electrical machine, $T_{\mathrm{en}}$. 


$$
\begin{aligned}
x_{1} & =\theta_{m} / i_{t} i_{f}-\theta_{w}, x_{2}=\dot{\theta}_{m}, x_{3}=\dot{\theta}_{w}, \\
x_{4} & =C_{s c} \times V_{d c}, x_{5}=T_{i c}, x_{6}=T_{e m}
\end{aligned}
$$

To achieve the required aims of the controller, weighting matrices are introduced into the cost function for the inputs and outputs, and tuning allows a heavy penaity to be applied to deviations from the reference vehicle speed, and for large increments in the throttle demand, whilst applying lighter penalties for deviation of super-capacitor voltage and increments in electrical machine torque demand. Hence, simultaneous reductions in vehicle shuffle and throttle transients, can be achieved.

As a result of a wide ICE operating speed range when equipped with a conventional transmission, the electrical machine, if attached directly in place of the engine flywheel, will often be required to operate above base speed. Similarly ICEs, particularly those equipped with turbochargers, produce limited -torque at low engine speeds. Such limitations in available torque can readily be integrated into an MPC strategy as a look-up table of speed dependant constraints on torque.

\section{Results}

Fig.10, shows results from the modelled electric-hybrid vehicle drive-train, under MPC control. Fig.10(a) shows the resulting wheel speed and super-capacitor voltage, along with the set-point references. The reference trajectory for angular velocity is considered rather arbitrary in this case, and is chosen to demonstrate controller constraint handling.
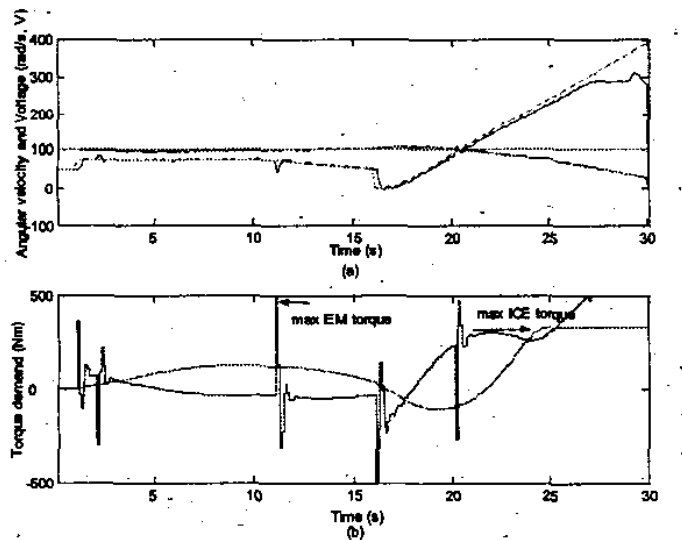

Fig. 10 Hybrid vehicle controller simulation

Fig.10(b) shows the control signals applied to the drive-train. The torque demand to the electrical machine can be seen to vary rapidly providing the transient torque required to control the drive-line oscillations, within the constraints of available toque, whilst the torque demand to the ICE is characterised by a slowly varying torque demand, again within available torque constraints. Notice also that whilst the super-capacitor voltage is allowed to vary, it is also operated within constraints.

\section{FUTURE RESEARCH}

The work is currently being enhanced by considering optimal, and adaptive, selection of cost function for the MPC formulation, and the ultimate incorporation of the resulting methodology on a hybrid-electric vehicle drive-train employing electrical torque assistance.

\section{REFERENCES}

[1] A. Balluchi, et.al. "Automotive engine control and hybrid systems: challenges and opportunities," Procedingsof the IEEE, Vol 88 , No. 7 , July 2000.

[2] T.J.J van den Boom. "Standard Predictive Control Toolbox" Delf University of Technology.

[3] E.F. Camacho, C. Bordons. "Model predictive control," Springer, 1999.

[4] P. Tondel, T.A. Johansen, A. Bemporad. "An algorithm for multiparametric quadratic programming and explicit MPC solutions," Tech. report 2001-7-W.

[5] S. Samuel, L. Austin, and D. Morrey, "Automotive test drive cycles for emission measurement and real-world emission levels a review". Proc Instn Mech Engrs Vol 216 Part D:J Automobile Engineering, 2002. pp. 555-564

[6] K Wipke, T. Markel, and D. Nelson, "Optimising Energy Management Strategy and Degree of Hybridization for Hydrogen Fuel Cell SUV'. EVS 18 Berlin, 2001.

[7] - U.S. EPA, "Final Technical Report in Aggressive Driving Behaviour for the Revised Federal Test Procedure Notice of Proposed Ruelmaking". EPA A-92-64.

[8] J.M. Maciejowski, "Predictive control with constraints", Prentice Hall, 2001.

[9] : D.W. Clarke, C. Mohtadi, P.S. Tuffs, "Generalised predictive control -part1, the basicalgorithm", Automatica, Vol.23 No.2, 1987. pp.137-148

[10] R.W. Weeks, J.J. Moskwa, “Automotive engine modeling for real-time control using MATLAB ${ }^{\text {TM}} /$ SMULINK $^{\text {MM }}$ " SAE 950417 SAE Detroit, 1995.

[11] R. Bosch GmbH, "Automotive Handbook," Robert Bosch GmbH, $3^{\text {rd }}$ Edition, 1993.

[12] U. Kiencke, L.Nielsen, "Automotive control systems for engine, driveline and vehicle," Springer-Verlag, 2000 